\title{
Wirtschaftspolitik und Statistik
}

\author{
Helmut Kramer \\ Österreichisches Institut für Wirtschaftsforschung, Wien
}

\begin{abstract}
Zusammenfassung: Die Welt, in der die amtliche Statistik ihre Aufgaben wahrzunehmen hat, hat sich dramatisch verändert. Die strategische Position der amtlichen Statistik ist schwierig geworden:

Die Umorientierung der Wirtschaftspolitik seit den sechziger Jahren hat die Bedeutung zentraler Ergebnisse der amtlichen Statistik (wie etwa der Volkswirtschaftlichen Gesamtrechnung) für die heimischen Entscheidungsträger reduziert. Gleichzeitig erfordern die durch den Beitritt Österreichs zur Europäischen Union eingegangenen Verpflichtungen aber eine signifikante Leistungsausweitung der amtlichen Statistik. Der Widerstand der Respondenten wächst. Deregulierung und Liberalisierung erschweren in vielen Fällen die Arbeit der Statistik. Kennzeichen der neuen Wirtschaft wie neue Arten wirtschaftlicher Aktivitäten, neue Formen von Beschäftigungsverhältnissen und Arbeitszeitregelungen sind mit traditionellen Instrumenten schwierig in den Griff zu bekommen.
\end{abstract}

Ein erfolgreiches Bestehen der amtlichen Statistik in Österreich setzt voraus, daß die neue Führung bürokratische Hindernisse überwinden und eine Aufwertung der Tätigkeit der Anstalt - auch im Urteil der Öffentlichkeit erreichen kann.

\section{Einleitung}

Die amtliche Statistik hat schon bessere Zeiten gesehen. Hin- und hergestoßen von Ignoranz und Knausrigkeit der Politik, Geringschätzung der Medien (und damit auch weiter Teile der Öffentlichkeit) einerseits und periodischen, sisyphusgleichen Kräfteanspannungen (Volkszählung, Großzählungen, ESVG-Anpassung usw.), andererseits ist sie zu einem Aschenputtel der öffentlichen Dienste geworden. Ihre an sich interessanten Gesichtszüge erscheinen vom täglichen Puddeln staubig, sie gehören einem rackernden Körper, dessen Bewegungsapparat da und dort erkennbar an arthrosis bureaucratica laboriert. Die Stiefmutter entläßt sie nun auf Grund eines neuen Reichsgesetzes, weniger weil das Kind längst über Alter und Reife verfügt, sondern weil es auch ihr finanziell weniger gut geht, in eine Art Selbständigkeit. Hier endet die Parallele zum Märchen: statt eines Königssohns nahen deren zwei. Daher ist auch nicht leicht anzugeben, wie das Happyend aussehen wird.

Der größere Teil der Probleme, mit denen die amtliche Statistik in Österreich in jüngster Zeit zu kämpfen hatte, ähneln denen in vergleichbaren, namentlich westeuropäischen Ländern. Sie sind Ausfluß gemeinsamer gesellschaftlicher, politischer und wirtschaftlicher Entwicklungen, die immer rascher und immer radikaler ein Überdenken althergebrachter Funktionen und Dogmen gebieten.

Ein Teil ist, das kann nicht übersehen werden, hausgemacht: letztlich von der österreichischen Politik, der die amtliche Statistik dienen soll und will, weil die Politik auch 
das, was im engeren Sinn im Amtsgebäude an der Hinteren Zollamtsstraße verursacht worden sein mag, geschehen ließ und daher verantwortet.

Die folgenden Überlegungen konzentrieren sich auf die allgemeinere, die europäische Situation der amtlichen Statistik und schließen nur gegen Ende auch solche an, die sich auf die hausgemachte Situation beziehen. Es sind Überlegungen eines Statistik-Konsumenten, der aus beruflichen Gründen auf Umfang und Qualität des für ihn unerläßlichen Rohstoffs Statistik angewiesen ist. Dabei stehen wirtschafts- und sozialpolitische Fragen im Vordergrund, nicht die Verwendung für unternehmerische und auch nicht die für andere als die genannten politischen Entscheidungen.

Die Welt, in der die amtliche Statistik ihre Aufgaben wahrzunehmen hat, hat sich in vieler Hinsicht dramatisch verändert. Die wichtigsten Veränderungen sind bei der Einschätzung der Staatsaufgaben, den Strukturen der Wirtschaft und den technischen, gesellschaftlichen und rechtlichen Abläufen der Statistik eingetreten.

\section{Staat und Statistik}

Der moderne Staat, zu dem sich bei genauem Hinsehen auch Österreich entwickelt, besser: entwickeln muß, verfolgt zwar letztlich die gleichen Ziele wie jener vor zwanzig oder dreißig Jahren, doch haben sich seine Kompetenzen, Aufgaben und Mechanismen erheblich verändert. Insbesondere ist sein umfassender Wohlfahrts- und Fürsorgeanspruch gegenüber der Bevölkerung zurückgedrängt worden, weil sich sein Versagen in vielen Bereichen herausgestellt hat. Die politische Reihung der Prioritäten hat sich aus wirtschaftlichen und politischen Gründen verschoben, und die Auffassungen, wie der Staat seine Ziele anstreben soll, präsentieren sich auf Grund des Eintritts in eine neue Phase der ideologischen und der wirtschafts- und sozialwissenschaftlichen Dialektik ziemlich grundlegend verändert. Darüberhinaus ist Österreich mit seinen westeuropäischen Partnern heute in das System der europäischen Integration voll eingebunden.

In der Wirtschaftspolitik ist das bis in die sechziger Jahre vorherrschende Modell der neoklassisch-postkeynesianischen Synthese unter dem Eindruck seiner Mängel schrittweise demontiert worden. Es hat einem längeren Suchprozeß Platz gemacht, der jetzt allmählich in eine neue eklektische Synthese zu münden scheint. Monetarismus, radikale Neoklassik, new macro-economics sind sich zumindest über die Unwirksamkeit und Unwünschbarkeit konjunktursteuernder Eingriffe einig. In der vergröbernden politischen Debatte sind dabei auch brauchbare Elemente des früheren Modells über Bord gegangen.

Die alte, ziemlich sterile Wachstumstheorie wurde durch new growth economics auf eine höhere Stufe der Unerklärlichkeit des Wachstums gehoben, indem der technische Fortschritt als Erklärung etwas mechanistisch ,endogenisiert“ wurde.

Weil auf diese Weise jedenfalls die staatliche Wirtschaftspolitik - nicht so die Notenbanken - nahezu ohne kurzfristig vorzeigbare Leistungsausweise geblieben wäre, bzw. diese sogar durch Absenz von „Aktionismus“ zu belegen gehabt hätte, was eine demokratiepolitisch bedenkliche Situation heraufbeschwört, verlegte sie sich stärker auf Instrumente der direkten Beeinflussung der mikroökonomischen Einzelentscheidungen auf der Angebotsseite (,Standortpolitik“, „Technologie-, Gründungs-, Infrastruktur- und Exportoffensiven").

Das immer drängender werdende Problem der Unterbeschäftigung in den westeu- 
ropäischen Ländern führte zwar noch keineswegs zu einigermaßen kongruenten Diagnosen und Therapien, jedoch zunehmend zu einer Erschütterung der monetaristischen und neoklassischen Dogmen. Tatsächlich fährt die Wirtschaftspolitik derzeit, sowohl in Österreich - das sich keine signifikant abweichenden Modelle mehr leisten kann - und in den meisten westeuropäischen Partnerländern einen pragmatischen Eklektizismus: monetaristische Grundregeln mit aktionistischem Aufputz bei insgesamt starkem Abbau der früheren direkten Einflußmöglichkeiten auf das Wirtschaftsgeschehen.

Gleichzeitig mit den Sorgen über die Beschäftigungslage werden die Konsequenzen globaler Wirtschaftsprozesse immer deutlicher, vielfach werden diese als Ursache jener angeprangert, so daß Bedarf an globaler Orientierung enorm gewachsen ist, vor allem im Finanzsektor.

\section{Was folgt daraus für die Wirtschaftsstatistik?}

Die volkswirtschaftliche Gesamtrechnung, jener frühe geistige Sproß des Keynesianismus, wird als Orientierungshilfe zwar nicht aufgegeben, aber ist doch gegenüber ergänzenden und konkurrierenden Datenbasen etwas in den Hintergrund gerückt. Auch als konzeptueller Bauplan der gesamten Wirtschaftsstatistik hat sie weitgehend ausgedient.

In den Vordergrund getreten sind alle Daten, die für die wie immer argumentierte geldpolitische Analyse benötigt werden: Geldmengenaggregate, Zinsen, gesamtwirtschaftliche Finanzierungsrechnung, Geldvermögens- und Verpflichtungsstrukturen, aber auch Preisentwicklungen. Der Verbraucherpreisindex wurde ins Zentrum der instrumentalen Orientierung der Makropolitik gerückt (inflation targeting). Seine Beschränktheit wurde unlängst peinlich bewußt, als sich herausstellte, daß auch von Vermögenspreisentwicklungen (asset prices) inflationäre und andere Impulse auf das gesamtwirtschaftliche Geschehen ausstrahlen.

Stark zugenommen hat aber auch die Beschäftigung mit den Bestimmungsgründen einzelwirtschaftlicher Entscheidungen, vor allem im Sektor der Produzenten. Bestimmungsgründe der Investitions-, Innovations- und Forschungsentscheidungen, durchaus nicht nur Finanzierungskosten, sondern auch Erwartungshaltungen und Strategien wurden interessant. Auch die Beschäftigungspolitik und die Sozialpolitik setzen stärker als früher auf der Individualebene an, durch immer neue Versuche mit aktiver Arbeitsmarktpolitik und mit besser gezielten Sozialprogrammen.

$\mathrm{Daß}$ bei all diesen, teilweise gut gerechtfertigten ,neuen“ Ansätzen der Makrozusammenhang verlorengehen kann, ist keine abstrakte Befürchtung. In Österreich werden die Sozialpartner ${ }^{1}$ nicht müde, darauf hinzuweisen, daß wirklich befriedigende Resultate bei der Bekämpfung der Arbeitslosigkeit die Vermeidung von makroökonomischen Fehlsteuerungen und Vorurteilen voraussetzen.

Für diese Art von Wirtschaftspolitik liefert die herkömmliche Wirtschaftsstatistik nicht wesentlich mehr als Rahmendaten. Die heroische Abstinenz der Wirtschaftspolitik von Konjunktursteuerung hat wahrscheinlich dazu geführt, daß auch Konjunkturdaten gegenüber früher nur recht verspätet und immer weniger verläßlich angeboten werden. Das kann sich jederzeit rächen.

\footnotetext{
${ }^{1}$ Beirat für Wirtschafts- und Sozialfragen: Wirtschaftspolitische Handlungsspielräume, Nr. 73, Wien 1998.
} 
Ergänzt wird dieses traditionelle Rahmenwerk von ad hoc hinzugefügten Bausteinen und Bauteilen der amtlichen Statistik, viel mehr als früher jedoch von nicht-amtlichen Befragungen aller Art, meist ohne ausreichendes wissenschaftliches Konzept, die sich untereinander und der amtlichen Statistik das Leben schwerer machen.

Freilich ist nicht zu anzunehmen, daß die amtliche Statistik an realitätsnahe Daten in heiklen Bereichen herankäme: Umsatz- und Gewinndaten, Bestimmungsgründe unternehmerischer Strategien, steuerlich relevante Leistungsdaten, Kriterien der Inanspruchnahme sozialpolitischer Leistungen und nicht zuletzt alle Phänomene des wahrscheinlich gewachsenen ,grauen Sektors“ der Wirtschaft, vor allem der „Pfusch“, ließen kaum wahrheitsgemäße Antworten auf amtliche Erhebungen erwarten.

Die wirtschaftspolitischen Schwerpunkte Geld- und Beschäftigungspolitik haben gerade in Österreich dazu die Bedeutung der Erhebungen und Auswertungen der Nationalbank einerseits und jene der Sozialpolitik, vor allem des Hauptverbands der Sozialversicherungsträger andererseits, im System der Statistik enorm aufgewertet.

Die staatliche Wirtschaftspolitik hingegen entnimmt der wirtschaftstheoretischen Debatte ebenso wie der Beobachtung von Globalisierungstendenzen, daß Österreich als „Standort“ wettbewerbsfähig bleiben müsse. Theoretisch ist das stark umstritten. Aber politisch ist dieser Ansatz griffig und plausibel. Und so sucht sie nicht ohne Enttäuschungen nach Indikatoren, die die „Performance“ Österreichs vor allem in ,struktureller“ Hinsicht beleuchten sollen.

Da sich solche eher ausnahmsweise plastisch aus amtlichen Erhebungen entnehmen lassen - sie entsprechen ja nur wenig dem alten makroökonomischen Leitbild der sechziger Jahre, dem der Ausbau der amtlichen Statistik auch noch 15 Jahre später gefolgt ist - bietet die Zeile für Österreich in internationalen Vergleichen (OECD ebenso wie EU) dieser Art mit betrüblicher Regelmäßigkeit die Feststellung n.a. Außerdem deckt die Regierung ihren Bedarf an Indikatoren im Rahmen der ,Strukturberichterstattung“ der frühen achtziger Jahre und der „Standortberichterstattung“ der neunziger Jahre bei den Wirtschaftsforschern, die dazu äußerst heterogene Informationsquellen zusammentragen.

\section{Europa und die Statistik}

Der Beitritt Österreichs zur Europäischen Union bedeutet für die amtliche Statistik gewissermaßen Gegenschub gegen die Tendenz zunehmenden staatlichen Agnostizismus. Nicht nur mußten die Erhebungskriterien, -rhythmen und -methoden angepaßt werden, was an sich mit enormem Aufwand verbunden war, es mußten auch sehr bedeutende neue Erhebungen und Datenbestände aufgebaut werden. Dabei hat sich herausgestellt, daß der Ausbaugrad der amtlichen Statistik in Österreich nur in einzelnen Segmenten den geforderten EU-Standards genügte.

Paradoxerweise führte dies, obwohl die oben geschilderten wirtschaftspolitischen Tendenzen durchaus auch in den anderen EU-Ländern vorherrschen, zu einem Quantensprung beim regelmäßigen Erhebungsumfang.

Die strategische Position der amtlichen Statistik wurde damit doppelt unerquicklich: nicht nur erschien ihre Bedeutung den heimischen Entscheidungsträgern infolge der zeitgeistigen Anschauungen über Wirtschaftspolitik weitgehend reduziert bis entbehrlich, sondern die EU-Verpflichtungen forderten gleichzeitig sogar eine signifikante Leistungs- 
ausweitung. Dies zwar auch in traditionellen Bereichen der Wirtschaftsstatistik und in der Volkswirtschaftlichen Gesamtrechnung, noch deutlicher jedoch in den Bereichen gemeinschaftlicher Wirtschaftspolitik: voran in der Agrarpolitik und dementsprechend der Agrarstatistik, aber auch in der Verkehrs-, Energie-, Regional-, Technologie-, Forschungsund nicht zuletzt Umweltpolitik.

Eine neue Qualität der europäischen Zusammenarbeit wurde 1999 mit der Wirtschaftsund Währungsunion erreicht. Gestützt auf den Vertrag von Maastricht wurde das System europäischer Zentralbanken etabliert, das augenblicklich einen enormen Bedarf an sehr präzisen, rezenten und detaillierten Daten entwickelte. Diesem konnten auch die regelmäßigen Kompilationen von EUROSTAT nur beschränkt genügen und so verlagerte sich die Verantwortung für amtliche Daten noch mehr als mit dem beschriebenen geldpolitischen Paradigmenwechsel zur Nationalbank, mit der symmetrischen Folge des relativen Bedeutungsverlustes von ÖSTAT.

\section{Liberalisierung, Privatisierung und Statistik}

Der Verband der österreichischen Gemeinwirtschaft bestellte bei ÖSTAT noch bis vor kurzem eine Sonderzusammenstellung der öffentlichen Wirtschaft in Österreich. Darin wurden der Staatssektor, die öffentlichen Betriebe, die verstaatlichten Unternehmungen und sogar die Genossenschaften und andere non-profit-Institutionen zusammengefaßt.

Diese Fleißarbeit wurde zwar noch 1998 (für das Jahr 1995) wieder vorgelegt, doch dürften dafür eher ideologische Gründe und das Vorhandensein von Finanzmitteln ursächlich gewesen sein, als die Erwartung irgendeiner sinnvollen Erkenntnis. Was ist heute noch eine verstaatlichte Bank? Was ein verstaatlichtes Industrieunternehmen? Fühlen sich diese, wenn es sich noch geben sollte, tatsächlich mit dem Genossenschaftssektor (Geld- und Warengenossenschaften) näher verwandt? Die Publikation wird wohl der letzte Nachhall der gemischten Wirtschaft gewesen sein, die Österreich einmal charakterisiert hat. (Jeder Beobachter weiß, daß es in Österreich noch politischen Einfluß auf manche dieser Bereiche gibt, doch er vollzieht sich in einer Weise, die mit der alten Definition der Gemeinwirtschaft und der Eigentums- oder Rechtsform nicht ausgelotet werden kann).

Die Privatisierung ist auch in Österreich ziemlich fortgeschritten. Die relevanten Daten interessieren die Börse und werden an der Börse bewertet, doch die amtliche Statistik bietet sie ganz sicher nicht.

Sie kann auch nicht bieten, was die staatliche Aufsicht über die deregulierten und zumindest teilweise privatisierten Sektoren benötigt, um ihren Kontrollansprüchen gerecht zu werden. Die Telekom-, die Elektrizitäts- und die Eisenbahnaufsichtsbehörden müssen, ähnlich wie die Bankenaufsicht, ihre Daten gefälligst selbst erheben. Und ähnliches gilt weitestgehend für die österreichische und europäische Wettbewerbsaufsicht in Bezug auf Kartelle und andere marktbeherrschende Stellungen und in Bezug auf Fusionen. Die amtliche Statistik kann dafür im besten Fall Rahmendaten, kaum je die Abgrenzung der relevanten Märkte und die Leistungsdaten (Preise, Kostenkalkulationen) zur Verfügung stellen. Das kann sie übrigens, ebenso wie bei den Regulierungsbehörden, auch in anderen europäischen Ländern weitgehend nicht. 


\section{Neue Wirtschaft und Statistik}

Die österreichische Wirtschaft befindet sich im Generalumbau, der die relativ stabilen Strukturen der Nachkriegsjahrzehnte zur Geschichte werden läßt. Fast nichts davon bleibt übrig. Nahezu siebzig Prozent der Beschäftigten sind heute im Dienstleistungssektor tätig, noch mehr in Dienstleistungsberufen, die einstmals vorherrschenden Ganztags-, Alleinverdiener-, Vollerwerbs- und Lebensberufs-Beschäftigungsverhältnisse beginnen in die Minderheit zu geraten gegenüber Teilzeit, Mehrfacharbeitsplätzen, häufigem Job- und Berufswechsel und atypischen Beschäftigungsverhältnissen.

Arbeitsrecht, Sozial- und Fiskalpolitik suchen zumindest minimale Legalisierung in diese Landschaft zu bringen. Die amtliche Statistik kann durch den Mikrozensus ein wenig in diese hinein-, aber sie bei weitem nicht ausleuchten. Die zunehmende Praxis, Anstellung durch Werkverträge oder durch noch weniger formelle Vereinbarungen zu ersetzen, hätte auch wenig Hoffnung auf realistische Respondenz.

Eine alte Erfahrung der Statistik ist, daß sie das Zählen von commodities und von hardware, auf deutsch von Äpfeln, Birnen, Ziegeln, Rohstahl und Kilowattstunden besser beherrscht als das von Beratungsgesprächen in der Bank und von Unterrichtsqualität in den Schulen. Wenn mehr als zwei Drittel der Wertschöpfung von solchen Tätigkeiten bestimmt werden, reduziert sich automatisch die Aussagefähigkeit der amtlichen Statistik.

Der rasche Strukturwandel in der Produktionsstruktur der österreichischen Wirtschaft wirft die alte Frage an die Statistik auf, ob sie im Interesse der Vergleichbarkeit über längere Zeiträume die Klassifikationen eher selten anpaßt, oder im Interesse der Aktualität wesentlich häufiger. Das, was in Amerika new economy bezeichnet wird, die dort angeblich einen signifikant höheren Wachstums- und Produktivitätstrend trägt, gibt es verständlicherweise nicht in der österreichischen Statistik.

$\mathrm{Da}$ wir der Informationsgesellschaft entgegengehen, haben Weitsichtige allerdings schon in den fünfziger und sechziger Jahren geweissagt. Jetzt sind wir eine solche, aber die Statistik kennt nach wie vor die alten Fachverbände, Innungen einerseits und ÖNACEKlassen andererseits. Das ist nicht ihre Schuld. Denn diese Klassifikationen sind ihr vorgegeben. Eine Sonderauswertung wäre wahrscheinlich möglich - und gefragt - aber, im Gegensatz zur Gemeinwirtschaft - hat die Informationswirtschaft halt noch keinen Verband.

Die neuen Verhältnisse auf den Märkten mit erhöhtem Wettbewerbsdruck, shareholder value-Vorgaben und Ausfall staatlicher Subventionen haben auch dazu beigetragen, daß die Unternehmen als Respondenten zunehmend störrisch geworden sind.

Nicht umsonst titelte das Organ der österreichischen Industrie im letzten Sommer „Controller an die Macht“. So sehr Controller sich die Verbesserung der internen Datenlage von Unternehmen zur Aufgabe machen, so sehr sind sie kaum dafür zu gewinnen, daß das Unternehmen Aufwand mit der Beantwortung von amtlichen und nichtamtlichen Erhebungen treibt. Und sie geben diesen Druck an die Unternehmensleitung und diese an die Kammer weiter. Die gerät in die leicht schizophrene Lage, daß sie diesen hautnahen Interessen ihrer Mitglieder Nachdruck verschaffen, gleichzeitig aber auch ihren eigenen wirtschaftspolitischen Informationsinteressen genügen müßte. 


\section{Österreich und amtliche Statistik}

Die angeführten Problembereiche sind nur ein Ausschnitt aus der generellen Problematik, in der sich die amtliche Statistik in den meisten europäischen Ländern, jedenfalls aber in Österreich derzeit befindet. Die weiteren Kapitel wären unter die Überschriften „Die Statistik und die Umwelt“, „Die Statistik und die ADV“ (wie das im Amtsdeutsch heißt), „Die Statistik und das Internet“ oder „Die Statistik und der Datenschutz“ zu stellen. Einerseits scheinen mir da teilweise die bisher erfolgten Schritte vergleichsweise erfolgreicher - Österreich verfügt etwa über eine Umweltstatistik, die in einer Reihe von Aspekten, nicht in allen, die internationalen Standards deutlich übertrifft, andererseits fehlen mir da die Erfahrungen, schließlich in Bezug auf Datenschutz vertritt der Autor die öffentlichen Interessen der Wissenschaft gegen die sehr weitgehenden Schutzinteressen, nicht gegen die gerechtfertigten jedes einzelnen Individuums und Staatsbürgers, sondern gegen Datenschutz als übergeordnetes Prinzip.

Es bleiben einige Schlußbemerkungen zu den Perspektiven der amtlichen Statistik in Österreich. Der Gesetzwerdungsprozeß des neuen Bundesstatistikgesetzes ging von äußerst unzulänglichen Beamtenentwürfen aus. In der Endphase wurde er massiv durch Fachwissen angereichert und erhielt so durchaus akzeptable und sogar fortschrittliche Eigenschaften neben solchen, die nach wie vor fragwürdig erscheinen.

Ein erfolgreiches Bestehen in der für das ÖSTAT sehr schwierig gewordenen Umgebung setzt voraus: daß die neue Führung des Amtes die bürokratischen Hindernisse für eine Umstrukturierung und Aufwertung der Tätigkeit des Amtes zu überwinden in die Lage gesetzt wird, möglichst, bevor die kleine Gruppe hervorragender Experten, die unverdrossen dort ihren weitgehend unbedankten Dienst tun, vollends entmutigt wird. Dies setzt zweitens voraus, daß trotz der formellen Distanz, die zwischen der Regierung und der Anstalt eingezogen wird, sich zumindest die zuständigen Regierungsmitglieder für das öffentliche Gut Statistik und für die neue Anstalt etwas mehr Interesse und Unterstützung aufbringen. Dazu wäre drittens eine weniger stereotype Pauschalaburteilung durch die Medien, als dies in den letzten Jahren dort Standard geworden ist, hilfreich. Schließlich scheinen mir Allianzen zwischen der Anstalt und anderen Statistikproduzenten einschließlich der Meinungsbefrager und Marktforscher, anzustreben, um auf die eine oder andere Weise die reichlich fragmentarische Landschaft der heutigen amtlichen Statistik zu konsistenteren und kompletteren Bildern zusammenzufügen.

Adresse des Autors:

Prof. Dr. Helmut Kramer

Österreichisches Institut für Wirtschaftsforschung

Postfach 91

A-1103 Wien, Österreich

Tel.: +4317982601210

Fax: +4317989386

Email: Helmut.Kramer@wifo.ac.at 
